\title{
Back Contact Effects on the Electro- Optical Properties of CdTe/CdS Solar Cells
}

D.H. Levi, D.S. Albin, and T.A. Gessert

National Renewable Energy Laboratory

L.M. Woods

Department of Electrical Engineering

Colorado State University, Ft. Collins, CO

Presented at the National Center for

Photovoltaics Program Review Meeting

Denver, Colorado

September 8-11, 1998

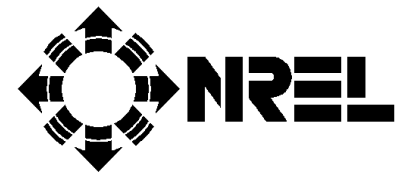

National Renewable Energy Laboratory 1617 Cole Boulevard

Golden, Colorado 80401-3393

A national laboratory of the U.S. Department of Energy Managed by Midwest Research Institute for the U.S. Department of Energy under contract No. DE-AC36-83CH10093

Work performed under task number PV903101

October 1998 


\section{NOTICE}

This report was prepared as an account of work sponsored by an agency of the United States government. Neither the United States government nor any agency thereof, nor any of their employees, makes any warranty, express or implied, or assumes any legal liability or responsibility for the accuracy, completeness, or usefulness of any information, apparatus, product, or process disclosed, or represents that its use would not infringe privately owned rights. Reference herein to any specific commercial product, process, or service by trade name, trademark, manufacturer, or otherwise does not necessarily constitute or imply its endorsement, recommendation, or favoring by the United States government or any agency thereof. The views and opinions of authors expressed herein do not necessarily state or reflect those of the United States government or any agency thereof.

Available to DOE and DOE contractors from:

Office of Scientific and Technical Information (OSTI)

P.O. Box 62

Oak Ridge, TN 37831

Prices available by calling 423-576-8401

Available to the public from:

National Technical Information Service (NTIS)

U.S. Department of Commerce

5285 Port Royal Road

Springfield, VA 22161

703-605-6000 or 800-553-6847

or

DOE Information Bridge

http://www.doe.gov/bridge/home.html 


\title{
Back Contact Effects on the Electro-Optical Properties of CdTe/CdS Solar Cells
}

\author{
D.H. Levi, L.M. Woods, ${ }^{*}$ D.S. Albin and T.A. Gessert \\ NREL, 1617 Cole Blvd., Golden, Colorado 80401 \\ ${ }^{*}$ Department of Electrical Engineering, Colorado State University, Fort Collins, CO 80523
}

\begin{abstract}
Studies of junction photoluminescence (PL) in CdTe/CdS solar cells reveal that backcontact application produces a dramatic qualitative change in the junction picosecond-PL spectrum. Prior to contact application, the spectrum has two peaks at energies of $1.501 \mathrm{eV}$ and $1.457 \mathrm{eV}$, corresponding to recombination in regions of $\mathrm{CdTeS}$ alloy with $2 \%$ and $12 \%$ sulfur content, respectively. After contact application, the spectrum consists of a single broad peak at $1.48 \mathrm{eV}$. Previous studies have shown that the nitric-phosphoric (NP) etch used in the contact procedure produces a layer of elemental tellurium (Te) on the CdTe surface. We postulate that the change in the near-junction PL spectrum is caused by a grain-boundary field effect due to perturbations of the grain-boundary conductivity and Fermi level.
\end{abstract}

\section{INTRODUCTION}

A critical issue in the production of thin-film $\mathrm{CdTe} / \mathrm{CdS}$ photovoltaic (PV) devices is the ability to form low-resistance ohmic contacts to p-type CdTe. Various surface treatments are applied prior to deposition of a metallic contact. One of the most effective is the use of a nitric-phosphoric (NP) acid etch [1]. It has been shown that the NP etch preferentially etches grain boundaries, and that this etch produces a layer of elemental Te on the surface of the CdTe grains $\left[^{2}\right]$. Issues of how the back contact affects the stability of $\mathrm{CdTe} / \mathrm{CdS} \mathrm{PV}$ modules have recently arisen. In light of the key role played by the back contact, it is important to further our understanding of how these contacts work and how back-contact treatments affect the CdTe material itself. Measurements of photoluminescence spectra on close-spaced sublimated (CSS) CdTe/CdS devices grown at NREL have found that the application of a back contact produces a significant qualitative change in the junction PL spectrum. This study examines the nature and causes of these changes.

\section{EXPERIMENTAL}

Typical room-temperature PL measurements on polycrystalline CdTe (px-CdTe) are dominated by recombination at defects because of the high density of defect states relative to 
the photoexcited carrier density. The PL spectra described in this study are unique in that a cavity-dumped dye laser is used as the excitation source. The laser provides pulses 5 picoseconds long at a repetition rate of $1 \mathrm{MHz}$. Due to the extremely low duty cycle of the laser $\left(5 \times 10^{-4} \%\right)$, we are able to inject much higher photoexcited carrier densities than is practical with a continuous-wave $(\mathrm{CW})$ laser. Initial carrier densities immediately after the laser pulse are approximately $4 \times 10^{16} \mathrm{~cm}^{-3}$. By comparison, typical densities for CW PL measurements are on the order of $2 \times 10^{13} \mathrm{~cm}^{-3}\left[^{3}\right]$. Hereafter, we will refer to these pulsed, high-excitation PL measurements as ps-PL. Under the high-injection conditions of the ps-PL experiment, one expects that the PL spectrum will be dominated by band-to-band transitions. We have observed that the ps-PL spectrum remains qualitatively unchanged, with an increase of up to 3 orders of magnitude in laser intensity. This confirms that the peaks observed in the ps-PL spectrum are due to band-to-band recombination.

In the experiments described in this paper, photoexcitation is through the transparent $\mathrm{CdS}$ window layer at a wavelength of $600 \mathrm{~nm}$. The 1/e penetration depth for this wavelength in CdTe is $0.2 \mu \mathrm{m}$. Numerical modeling indicates that more than $90 \%$ of the photoexcited carriers recombine within the first $3000 \AA$ of CdTe adjacent to the $\mathrm{CdTe} / \mathrm{CdS}$ interface, hence the term "junction photoluminescence." The measurement is conducted at $300 \mathrm{~K}$ under open-circuit conditions. Spectral resolution is $1 \mathrm{~nm}$.

We have studied the effect of numerous back-contact treatments on the observed ps-PL spectrum for the CdTe adjacent to the CdTe/CdS interface. Samples in this study consist of standard NREL CSS-CdTe / CBD-CdS / $\mathrm{SnO}_{2}$ / 7059 glass. The CdTe thickness is approximately $10 \mu \mathrm{m}$, with a grain size of about $3 \mu \mathrm{m}$. We have studied a matrix of backcontact treatments including NP etch only, NP etch followed by annealing, NP etch followed by $\mathrm{HgTe}$ /graphite paste, $\mathrm{HgTe}$ /graphite paste alone, then with annealing, application of silver conducting paint, and finally, evaporated Te. We have also studied the effects of using ionbeam milling to remove several microns of CdTe from the back surface of the sample after these treatments.

\section{PL RESULTS}

As discussed above, the conditions of our PL experiment measure band-to-band recombination spectra of an approximately 3000- $\AA$-thick layer on the CdTe-side of the CdTe/CdS interface. Fig. 1 illustrates the typical junction PL spectrum measured on NREL polycrystalline $\mathrm{CdTe} / \mathrm{CdS} / \mathrm{TCO}$ and $\mathrm{CdTe} / \mathrm{TCO}$ device structures. All deposition and treatment conditions are identical for the two films. The only difference is the presence of the CdS layer in one film. The differences in the two spectra are striking. The CdTe/TCO film spectrum consists of a single Gaussian peak at the 1.51-eV bandgap of CdTe. The $\mathrm{CdTe} / \mathrm{CdS} / \mathrm{TCO}$ film spectrum consists of two peaks that can be fit with a sum of two Gaussians. The fitting functions are illustrated by the dashed lines in Fig.1. 


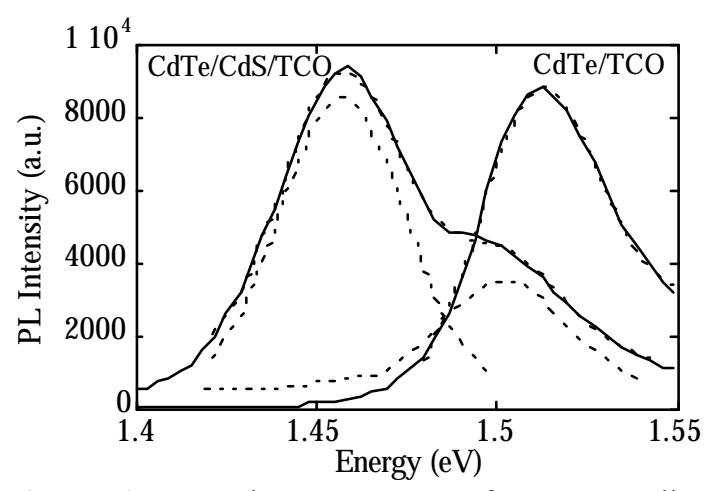

Figure 1. Junction PL spectra for pre-metalization devices illustrating effect of presence of $\mathrm{CdS}$ layer. Dashed lines demonstrate Gaussian fits.
The peak energies of the two peaks in the $\mathrm{CdTe} / \mathrm{CdS} / \mathrm{TCO}$ spectrum are 1.501 and $1.457 \mathrm{eV}$, respectively. The bandgap of a CdTeS alloy initially decreases as sulfur is added to CdTe, up to a sulfur concentration of about $20 \%$ [ $\left.{ }^{4}\right]$. A linear fit to the bandgap in this concentration regime gives a reduction of $4.5 \mathrm{meV}$ in bandgap for each $1 \%$ increase in sulfur concentration. We conclude that the two peaks in the CdTeS spectrum are due to regions of alloy with compositions of $2 \%$ and $12 \%$,

respectively. The $12 \%$ composition is consistent with grazing-incidence X-ray diffraction measurements of alloy composition on lift-off samples $\left[{ }^{5}\right]$, which probe only the first $50 \AA$ of the film. It is not clear at this time why the spectrum displays two distinct peaks rather than a single broad peak spanning the range of bandgaps from $12 \%$ to $0 \%$ sulfur. Possible explanations are an electric-field-induced segregation of photoexcited carriers, or a $\mathrm{CdTeS}$ alloy composition distribution that is bimodal instead of changing smoothly with distance from the interface.

The effect of contact application is illustrated in Fig. 2. The solid line shows the junction ps-PL spectrum prior to contact application, whereas the dashed line is for a sample after contact application. The post-contact spectrum consists of what appears to be a single broad peak at $1.48 \mathrm{eV}$. We have attempted to fit this peak with a wide variety of fitting functions. The asymmetry of the peak is such that the best fit is achieved using two Gaussians with peak energies of $1.509 \mathrm{eV}$ and $1.487 \mathrm{eV}$. The peak at $1.509 \mathrm{eV}$ is consistent with PL from px-CdTe, whereas the lower-energy peak matches the bandgap of a CdTeS alloy with about $3 \%$ sulfur.

The standard NREL method for applying back contacts is to first etch the back surface with a nitric-phosphoric acid etch. Then, a HgTe-doped graphite paste is applied, and the

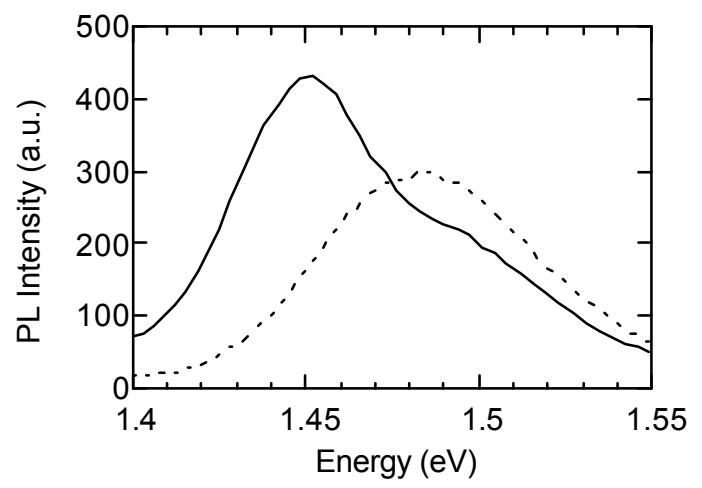

sample is annealed. Finally, silver paste is applied to form the metallic contact. By measuring ps-PL spectra at each step in this process, we found that the change in the spectrum is produced by NP etch without annealing. Measurements of psPL on the back surface of an etched $\mathrm{CdTe} / \mathrm{CdS}$ device showed a decrease in PL intensity, but no change from the single $1.509-\mathrm{eV}$ ps-PL peak observed prior to NP etch. This is good evidence

Figure 2. Junction ps-PL spectra for an NREL that the two-peak ps-PL spectrum on the $\mathrm{CdTe} / \mathrm{CdS}$ device structure before (solid line) and after junction side is associated with the (dashed line) contact application. 


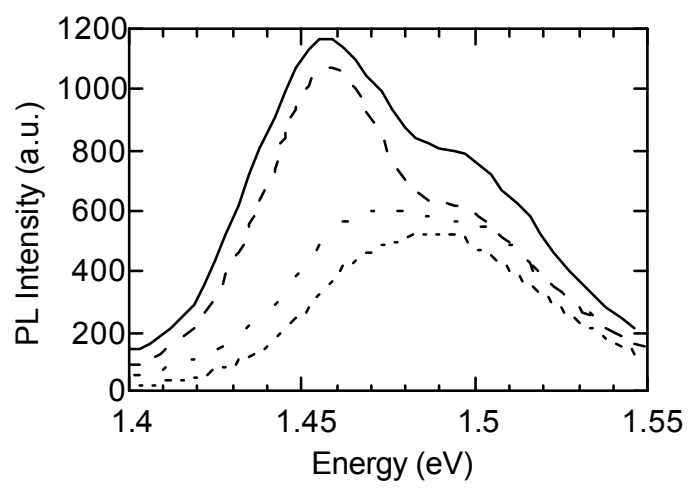

Figure 3. Junction ps-PL spectra showing evolution of spectra with increments of NP etch time. Etch times of $0,10,20$, and 30 seconds correspond to solid, large dash, medium dash, and smallest dashed lines. For clarity the intensities have been scaled to avoid overlap.

presence of CdTeS alloy and not just a defect level produced by the NP etch at the grain boundary.

As a further test of our interpretation of the post-NP etch spectrum being composed of two peaks, we have monitored the ps-PL as a function of incremental steps of NP etch time. The typical NP etch procedure for NREL $\mathrm{CdTe} / \mathrm{CdS}$ cells requires a 40 -second etch time. Fig. 3 presents ps-PL spectra for etch times of $0,10,20$, and 30 seconds. Beyond 30 seconds of etch time, there is very little change in the spectrum. It is clear from these spectra that the change in PL produced by the $\mathrm{NP}$ etch is not a binary, "on or off" effect. Rather, the shift from two peaks to one is gradual and is consistent with increasing thickness and depth of Te layers in the grain boundaries.

A study of NP etch effects on single-crystal CdTe has found that a 60-second NP etch removes cadmium, leaves $30 \mathrm{~nm}$ of elemental Te on the CdTe surface, and produces a transition region of Te-rich CdTe of $50 \mathrm{~nm}$ thickness $\left[{ }^{6}\right]$. It is known that Te is a degenerate p-type semiconductor with a bandgap of $0.33 \mathrm{eV}\left[{ }^{7}\right]$. The study of NP-etched CdTe surfaces also used evaporation to apply Te to the surface of px-CdTe and found that the Fermi level of Te layers less than $26 \AA$ thick was pinned by the midgap surface states of the CdTe grain, and thus, the layer was n-type. The Te layers greater than $76 \AA$ thick were degenerate p-type. The primary distinctions between NP etch and evaporated Te are that $\mathrm{NP}$ etch preferentially etches grain boundaries and produces a 50-nm-thick transition region of Te-rich CdTe, whereas evaporation produces an abrupt interface between Te and CdTe.

To determine whether or not the Te layer produced by the NP etch is responsible for the change of the ps-PL spectrum, we investigated several other back-surface treatments. Application of silver conducting paint to the back surface had no effect on the junction ps-PL spectrum. Bromine-methanol (Br-Me) is an etch for CdTe that is known to leave a Te-rich surface. Upon etching an NREL $\mathrm{CdTe} / \mathrm{CdS}$ device structure, we found that $\mathrm{Br}-\mathrm{Me}$ produces the same shift in the junction PL as that observed for NP etch. X-ray photoelectron spectroscopy (XPS) measurements confirm that the Br-Me etched surface is elemental Te. We also used vacuum evaporation to deposit a 50-nm layer of Te on the back surface of a $\mathrm{CdTe} / \mathrm{CdS}$ device structure and observed a change in the ps-PL spectrum identical to that observed for NP and Br-Me etches.

At this point, it is reasonable to conclude that the Te layer is causing the change in the psPL. The key question is whether the change is a grain-boundary effect or a back-surface field effect. The fact that evaporated Te produces the spectral shift would indicate that it is a back-surface field effect. We have used the device simulation program SimWindows to model the effects of a degenerate p-type layer on the back surface of a $\mathrm{CdTe} / \mathrm{CdS}$ device. 


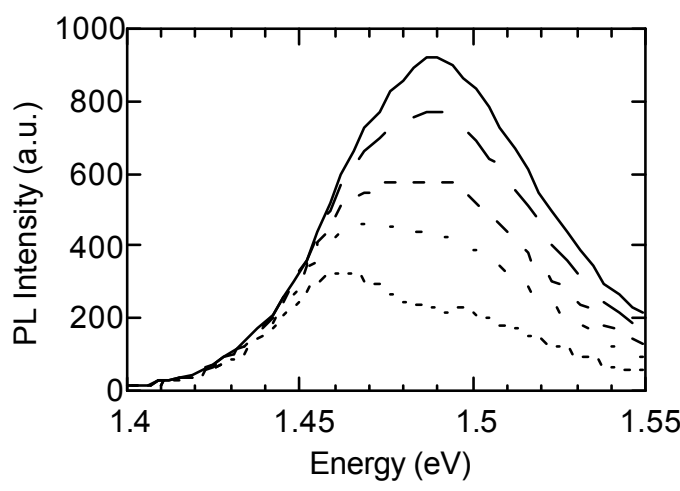

Figure 4. Evolution of the junction ps-PL spectrum with ion-beam milling depth. The five spectra in the figure are for milling depths of $0,0.18 \mu \mathrm{m} 0.72 \mu \mathrm{m}, 1.26$ $\mu \mathrm{m}$, and $2.6 \mu \mathrm{m}$. Smaller dashed lines correspond to increased milling time. For clarity the intensities have been scaled to avoid overlap.

In this numerical simulation, the backsurface field produced by a contact does not perturb photoexcited carrier distributions at the junction. This makes it unlikely that the observed effect is due to a back-surface field.

To further test this hypothesis, we have used ion-beam milling to remove the layer of Te from the back surface of an NP-etched sample. Fig. 4 shows ps-PL spectra for samples that have milling depths of $0,0.18,0.72,1.26$, and 2.6 $\mu \mathrm{m}$. As can be seen in the figure, and has been verified by peak fitting, the 0.18 $\mu \mathrm{m}$ spectrum is unchanged from the NP etched spectrum, whereas each successive step in milling changes the spectrum more and more toward that observed for non-etched samples. The 2.6- $\mu \mathrm{m}$ sample has a spectrum that matches that for a non-etched sample. XPS measurements of the ion-milled surfaces show that the $0.18-\mu \mathrm{m}$ sample is stoichiometric CdTe, whereas the longer milling times become increasingly $\mathrm{Cd}$-rich at the back surface.

The fact that the PL spectrum of the $0.18-\mu \mathrm{m}$ milled sample is unchanged from an NPetched sample confirms the results of our device modeling, namely, that the effect on the junction PL spectrum is not a back-surface field effect. The interpretation of the gradual return to a non-etched spectrum is more complicated. Such behavior with milling depth would be consistent with relatively thick layers of elemental Te in the grain boundaries near the back surface, with decreasing thickness moving toward the front of the device. Thick, degenerate p-type grain boundaries near the back surface, together with the highly conductive Te layer down the grain boundaries, could affect the Fermi level of grain boundaries near the junction region where the junction ps-PL originates. As the thick Te layers are removed from the back, the Fermi level of the grain boundaries again becomes pinned to the midgap surface states of the CdTe grain surface just as prior to NP etch.

\section{CONCLUSIONS}

We have provided evidence that the qualitative change in the junction ps-PL spectrum associated with the NP etch process is caused by the accumulation of elemental Te in the grain boundaries. How this perturbation of the PL spectrum comes about is not completely clear at this time. We can postulate a model of the recombination process that produces the two-peak PL spectrum and of how the presence of elemental Te in the grain boundaries could perturb the PL spectrum.

Generally accepted models hold that charged defects and impurities segregated at grain boundaries will produce a depletion layer at the grain boundary $\left[^{8}\right]$. The Fermi level in such 
a grain boundary will be near mid-gap. After the NP etch process, there will be a layer of elemental Te, which, if thick enough, will be degenerate p-type. This is a large perturbation of the position of the Fermi level and also a major increase in the conductivity of the grain boundary. These changes will in turn produce significant changes in the electric fields and carrier distributions in the near-grain-boundary regions of the interior of the grain.

Numerous studies have shown that interdiffusion across the CdTe - CdS interface produces a thin (200-300 nm) layer of CdTeS alloy on the CdTe side of the interface [ ${ }^{9}$. Recent energy-dispersive analysis measurements (EDS) have shown that the sulfur content within a CdTe grain near the $\mathrm{CdTe} / \mathrm{CdS}$ interface has a radial dependence, with the highest concentration at the grain boundary and a rapid decrease moving toward the center of the grain $\left[{ }^{10}\right]$.

This structural information brings us to a model for the ps-PL spectra that we have observed. Prior to the NP etch treatment, recombination occurs throughout the grain. After NP etch, the population of one or both carrier types is depleted near the grain boundary, and recombination shifts toward the center of the grain. The net effect is that the percentage of PL originating in pure CdTe will increase, whereas the average sulfur content of the CdTeS participating in recombination processes will decrease. Thus, the post-NP-etch PL spectrum should have a decrease in the amplitude of the lower-energy, CdTeS alloy peak, and this peak should shift toward higher energy. This is completely consistent with all of the observations reported in this study.

\section{ACKNOWLEDGEMENTS}

This work was supported by the U.S. Department of Energy under Contract No. DEAC36-83CH10093 with NREL. The authors would like to thank David Niles for XPS measurements and deposition of Te, and Mowafak Al-Jasim for insightful discussions regarding EDS and TEM measurements in CdTe.

\section{REFERENCES}

\footnotetext{
[1] Y.S. Tyan, U.S. Patent 4,319,069 (March 9, 1982).

$\left.{ }^{2}\right]$ X. Li, D.W. Niles, F.S. Hasoon, R.J. Matson, and P. Sheldon, submitted for publication, 1998.

$\left.{ }^{3}\right]$ The calculated densities assume a carrier lifetime of $500 \mathrm{ps}$.

$\left.{ }^{4}\right]$ D. Albin, et al., Fall 1997 Materials Research Society Symposium G, Boston, MA, p. 215.

$\left.{ }^{5}\right]$ ibid [4].

$\left.{ }^{6}\right]$ D.W. Niles, X. Li, P. Sheldon, and H. Hochst, Journal of Applied Physics, 77(9), 1995, p. 4489.

$\left.{ }^{7}\right]$ Vol. 17, Landoldt-Bornstein New Series, Numerical Data and Functional Relationships in Science and Technology, edited by K.-H. Hellwege (Springer, Berlin, 1983), pp. 106-117.

$\left.{ }^{8}\right]$ A.L. Fahrenbruch and R.H. Bube, Fundamentals of Solar Cells (Academic Press, New York, New York, 1983), p.387 \& ff.

$\left[{ }^{9}\right]$ B. E. McCandless and R. W. Birkmire, Solar Cells, 31, 1991, pp. 527.

$\left.{ }^{10}\right]$ Mowafak Al-Jassim, personal communication, Sept, 1997.
} 